\title{
THE SHEAL DIFFUSE X-RAY SPECTROMETER EXPERIMENT
}

\author{
W. T. Sanders, S. L. Snowden, and R. J. Edgar \\ University of Wisconsin-Madison, Madison, WI, USA
}

\begin{abstract}
The Diffuse X-ray Spectrometer (DXS) experiment is part of NASA's SHEAL 2 mission, scheduled to be flown as an attached Shuttle payload in 1992. The DXS is designed to measure the spectrum of the low energy $(0.15$ to $0.28 \mathrm{keV})$ diffuse $\mathrm{x}$-ray background with energy resolution better than $0.01 \mathrm{keV}$. This paper describes the DXS experiment and presents the results of calculations of the anticipated data.
\end{abstract}

\section{INTRODUCTION}

At energies less than $0.28 \mathrm{keV}$ the diffuse $\mathrm{x}$-ray background is thought to originate mostly in a local interstellar plasma with a temperature near $10^{6} \mathrm{~K}$, if collisional equilibrium is assumed (McCammon et al. 1983; Bloch et al. 1986). X-ray emission in this energy range from such a plasma is believed to be primarily in the form of collisionally excited lines of such ions as Si VIII, Mg IX, S VIII, and $\mathrm{Ne}$ IX, and the purpose of the Diffuse X-Ray Spectrometer (DXS) experiment is to search for and study those emission lines. It is important to obtain spectra of this diffuse emission, both to verify that it arises from a hot plasma, and to study the properties of that plasma. Such properties include the temperature, the relative abundances of the heavy elements responsible for the emission lines, and the ionization state of the gas. The plasma may not yet have reached ionization equilibrium if, for example, the local component of the emission is due to a blast wave with an age of order $10^{5} \mathrm{yr}$ (Cox and Anderson 1982; Edgar 1986; Rothenflug and Arnaud, this volume).

\section{THE DXS INSTRUMENT}

Figure 1 illustrates DXS in the Shuttle orbiter in the SHEAL 1 configuration and Table 1 lists some instrument parameters. The DXS employs a curved lead stearate Bragg reflector to disperse the $\mathrm{x}$-rays across the face of a one-dimensional position-sensitive proportional counter. Figure 2 presents a cross section of a DXS detector assembly and Table 2 lists some detector parameters. By having two detectors facing in complimentary directions and scanning back-and-forth across the same arc of the sky, complete wavelength coverage over the entire scan arc can be obtained. The effective DXS area solid angle product is greater than $0.01 \mathrm{~cm}^{2} \mathrm{sr}$ from $0.15 \mathrm{keV}$ to $0.27 \mathrm{keV}$, peaking near $0.019 \mathrm{~cm}^{2}$ sr around $0.18 \mathrm{keV}$. In a mission with four days devoted to the collection of data by DXS, it is possible to obtain 5000 seconds of prime low-background data in each detector at all wavelengths for each of ten $15^{\circ} \times 15^{\circ}$ regions along a $150^{\circ}$ arc. This should result in about 10,000 counts in each spectrum, which will allow useful spectral fitting for the individual regions. 


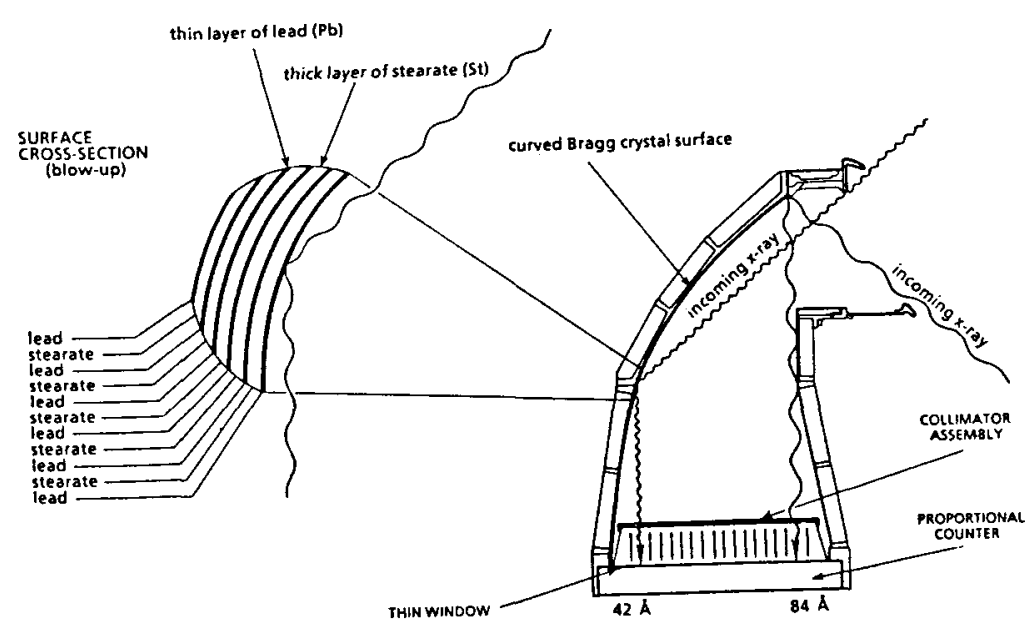

Figure 1. (below) DXS in the SHEAL 1 configuration. The DXS experiment consists of two instruments, one attached to each side of the Shuttle's cargo bay. Each DXS instrument is mounted on a SPOC (Shuttle Payload of Opportunity Carrier) plate that is attached to the Shuttle. The SPOC avionics delivers electrical power and ground commands to DXS and receives scientific and engineering data from DXS for transmission to the Operations Control Center. The arrows labeled "Diffuse X-ray Spectrometer" point to the rotating detector assemblies.

Figure 2. (above) A cross section of a DXS detector assembly. Incident $\mathrm{X}$-rays are Braggreflected from a cylindrical lead stearate crystal panel and enter a collimated proportional counter that records the $\mathrm{x}$-ray position along the direction of dispersion. Because a particular element of a counter sees only a limited piece of the crystal panel, it receives only those $\mathrm{x}$-rays that are Bragg-reflected at a particular angle. At any given time, different elements of the counter along the dispersion direction receive different energy $x$ rays from different parts of the sky. Rotation of a detector extends the spectral coverage of sky directions in the middle of the scanned arc in addition to simply observing more of the sky. Adding a second detector that mirrors the scanning motions of the first greatly increases the fraction of the scan path that receives full spectral

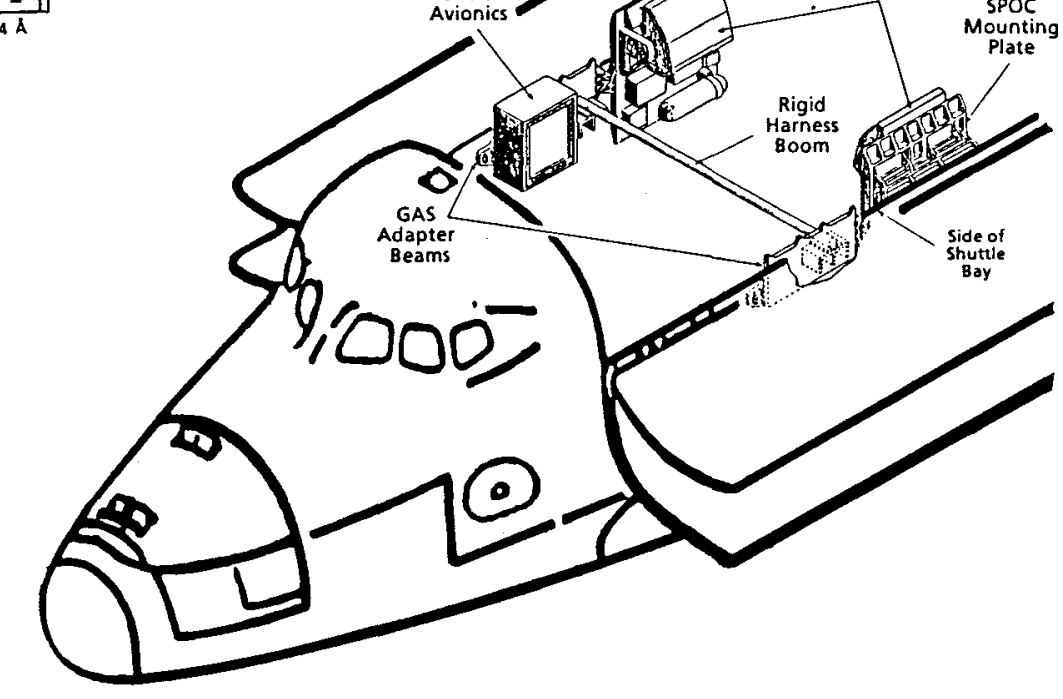
coverage. 

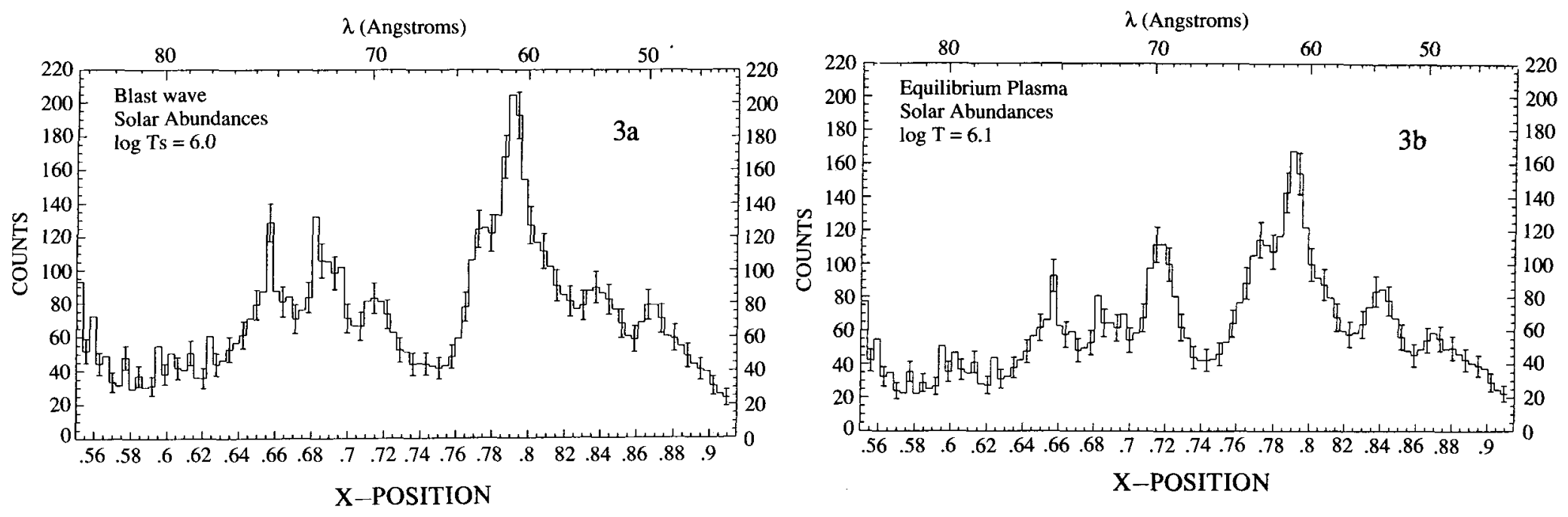

Figure 3. (a) Predicted spectrum of a blast wave (viewed from inside) with the log of the post-shock temperature equal to 6.0. (b) Predicted spectrum from an equilibrium plasma with $\log T=6.0$. (c) Predicted spectrum from an equilibrium plasma with $\log \mathrm{T}=6.1$.

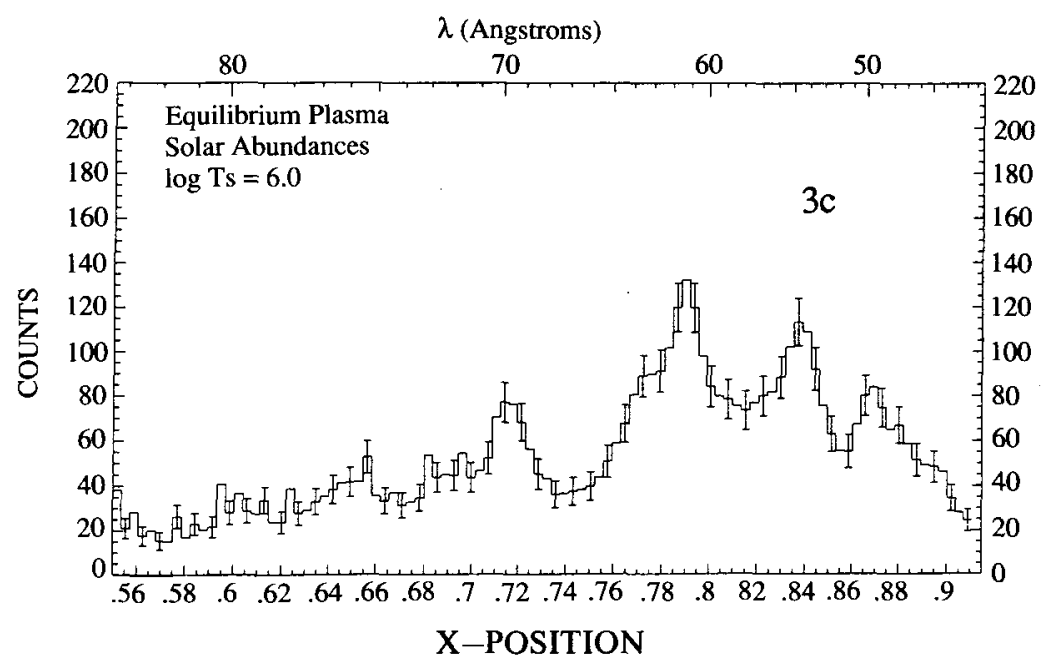


TABLE 1. Experiment Parameters

Number of Instruments:

Weight per instrument:

DC Power Consumption:

Size of SPOC Plate:

Region Scanned:

Observing Time per Resolution Element:

Total Good Observing Time:

Mission Operational Time:
TABLE 2. Detector Parameters

2 Crystal Panel Radius of Curvature: $63 \mathrm{~cm}$ 700 lbs Crystal Panel Dimensions: 14" x 24" 900 Watts 2d Spacing of Lead Stearate Crystals: $101 \AA$ $50^{\prime \prime} \times 60^{\prime \prime}$ Integrated Reflectivity of Crystals: $0.6 \mathrm{mr}$ Field of view: $\quad 15^{\circ} \times 15^{\circ}$ (FWHM) $15^{\circ} \times 150^{\circ} \quad$ Energy Resolution: $\quad \Delta \mathrm{E} \approx 10 \mathrm{eV}$ Proportional Counter Open Area: 9" x 21" Mesh Transmission: $\quad 68 \%$ $5000 \mathrm{~s}$ Thin Window Composition:

Formvar:

$60 \mu \mathrm{g} \mathrm{cm}^{-2}$ UV 24: $30 \mu \mathrm{g} \mathrm{cm}^{-2}$

\section{THE MODELS}

The blast wave spectrum shown in Figure 3a is that of a Sedov model with negligible external pressure. Ionization and recombination rates, as well as emission line parameters, are taken from the Raymond and Smith $(1977,1987)$ code. This allows for the gas to be in a non-equilibrium ion state, which can have profound effects on the resulting $x$-ray spectrum. Temperature effects can be seen by comparing Figures $3 b$ and $3 \mathrm{c}$, both of which assume an equilibrium plasma, with atomic physics again given by Raymond and Smith. Solar abundances are assumed, and the integration time in each case is 10,000 seconds ( 5000 seconds for each of two detectors). Each of the spectra also includes two absorbed components: one with $\log \mathrm{T}=6.5$ absorbed by a hydrogen column $\mathrm{N}_{\mathrm{H}}=1.0 \times 10^{20} \mathrm{~cm}^{-2}$, and a $11 \mathrm{E}^{-1.4}$ photons $\mathrm{cm}^{-2} \mathrm{~s}^{-1} \mathrm{sr}^{-1}$ spectrum, absorbed by $\mathrm{N}_{\mathrm{H}}=5.0 \times 10^{20} \mathrm{~cm}^{-2}$. These two absorbed components contribute approximately $10 \%$ of the predicted counts.

It is a pleasure to acknowledge the guidance of the Principle Investigator of the DXS experiment, W. L. Kraushaar, and the work of D. McCammon, who designed and built the DXS detectors. The engineering staff of the University of Wisconsin's Space Science and Engineering Center deserves particular mention for design, contruction and testing of the instrument. This work was supported by NASA contract NAS 5-26078.

\section{REFERENCES}

Bloch, J. J., Jahoda, K., Juda, M., McCammon, D., Sanders, W. T., and Snowden, S. L. 1986, Ap. J. (Letters), 308, L59.

Cox, D. P. and Anderson, P. R. 1982, Ap. J., 253, 268.

Edgar, R. J. 1986, Ap. J., 308, 389.

McCammon, D., Burrows, D. N., Sanders, W. T., and Kraushaar, W. L. 1983, Ap. J., 269, 107.

Raymond, J. C. and Smith, B. W. 1977, Ap. J. Supp., 35, 419. 1987, private communication.

Rothenflug, R. and Arnaud, M. 1988, this volume. 